INPLASY

PROTOCOL

To cite: Chai et al. The prevalence of mental health problems for Chinese children and adolescents during COVID-19 in China: A metaanalysis. Inplasy protocol 202150032. doi: 10.37766/inplasy2021.5.0032

Received: 08 May 2021

Published: 09 May 2021

Corresponding author: Jiabao Chai

chaijiabao1988@126.com

Author Affiliation:

Beijing Huilongguan Hospital, Peking University Huilongguan Clinical Medical School, Beijing, China.

Support: No financial support.

Review Stage at time of this submission: Formal screening of search results against eligibility criteria.

Conflicts of interest:

None declared.

\section{The prevalence of mental health problems for Chinese children and adolescents during COVID-19 in China: A meta-analysis}

\author{
Chai, J1; Xu, H2; Ning, A3; Zhang, P4; Liu, F5; He, S6; Hu, N7; \\ Xiao, $X^{8} ;$ Cui, Y9; Li, Y10.
}

Review question / Objective: Investigating the prevalence of mental health problems (depression and anxiety) among Chinese children and adolescents during COVID-19 in China. Condition being studied: The outbreak of coronavirus disease 2019(COVID-19) has caused mental health problems among the public and health care workers in China, especially for children and adolescents, a vulnerable group that might present with more mental problems. It seems that there is a rapid growth for the mental problems of Chinese children and adolescents during the outbreak of COVID-19. Although there were several studies reported the depression or anxiety for children and adolescents, but it showed mixed results. In present study, we aim to perform a meta-analysis to identify the confirmed prevalence of depression and anxiety for mental health in Chinese children and adolescents during the home confinement.

INPLASY registration number: This protocol was registered with the International Platform of Registered Systematic Review and Meta-Analysis Protocols (INPLASY) on 09 May 2021 and was last updated on 09 May 2021 (registration number INPLASY202150032).

\section{INTRODUCTION}

Review question / Objective: Investigating the prevalence of mental health problems (depression and anxiety) among Chinese children and adolescents during COVID-19 in China

Condition being studied: The outbreak of coronavirus disease 2019(COVID-19) has caused mental health problems among the 
public and health care workers in China, especially for children and adolescents, a vulnerable group that might present with more mental problems. It seems that there is a rapid growth for the mental problems of Chinese children and adolescents during the outbreak of COVID-19. Although there were several studies reported the depression or anxiety for children and adolescents, but it showed mixed results. In present study, we aim to perform a meta-analysis to identify the confirmed prevalence of depression and anxiety for mental health in Chinese children and adolescents during the home confinement.

\section{METHODS}

Search strategy: Six databases were searched, including PubMed, Web of Science, PsycINFO, Google, WangFang, CNKI.

Participant or population: Children and adolescents in China.

Intervention: This study was a crosssectional meta-analysis without intervention.

Comparator: This study was a crosssectional meta-analysis without comparator.

Study designs to be included: A metaanalysis will be carried out based on the data of published articles to calculate the prevalence of depression and anxiety in children and adolescents during the COVID-19 period.

Eligibility criteria: The literatures included in this study were all cross-sectional studies. Both the inclusion criteria and exclusion criteria were developed as follows: Inclusion Criteria: 1.the participants were Chinese children and adolescents; 2.the date of investigation was during COVID-19 home isolation; 3.validated mental health screening tools were used. Exclusion Criteria: 1.no data on the prevalence of mental problems were reported; 2.the assessment did not include the depression and anxiety symptoms; 3 . the age of participants was over 18 years old (such as college students).

Information sources: Six databases were searched, including PubMed, Web of Science, PsycINFO, Google , WangFang, CNKI. Participation studies are published after November 1, 2019。The search terms are: COVID-19, "children," "teens," mental health issues, depression, anxiety.

Main outcome(s): Investigated the specific prevalence of mental health problems (depression and anxiety) in Chinese children and adolescents during COVID-19.

Additional outcome(s): Recommend effective tools for measuring depression and anxiety in children and adolescents.

Data management: The two authors (Jiabao Chai and Huili $\mathrm{Xu}$ ) extracted and managed the data separately, including each study, and agreed on any differences.

Quality assessment / Risk of bias analysis: The quality of each included study was assessed by the Joanna Briggs Institute(JBI) Critical Appraisal Checklist for Studies Reporting Prevalence Data, which included nine items. The Cochrane Risk of Bias Tool consists of random sequence generation, allocation concealment, performance and detection bias, incomplete outcome data, selective reporting, and other bias.

Strategy of data synthesis: A total of 12 studies were identified.We extracted the following information from the included studies: authors, participants, mean ages, sample sizes, the number of boys and girls, the screening tools used, survey location.

Subgroup analysis: The effects of age and gender on the incidence of depression and anxiety were analyzed by subgroup analysis using age and gender as variables.

Sensitivity analysis: Sensitivity analysis will be conducted on the extracted data. 
Language: Chinese and English.

Country(ies) involved: China.

Keywords: Child and Adolescents, China, meta-analysis, Depression, Anxiety.

Contributions of each author:

Author 1 - Jiabao Chai The author drafted the manuscript.

Email: chaijiabao1988@126.com

Author 2 - Huili Xu - The author contributed to the development of the selection criteria, and the risk of bias assessment strategy.

Email: 44897777@qq.com

Author 3 - Ying Li - The author provided statistical expertise.

Email: yyjnly@126.com 